\title{
Towards a Dynamic Neuropharmacology: Integrating Network and Receptor Levels
}

\author{
Péter Érdi ${ }^{1}$ and János Tóth ${ }^{2, \star}$ \\ ${ }^{1}$ Center for Complex Systems Studies, Kalamazoo College, Kalamazoo, MI 49006, USA \\ and Department of Biophysics, Research Institute for Particle and Nuclear Physics of the \\ Hungarian Academy of Sciences \\ perdi@kzoo.edu \\ ${ }^{2}$ Department of Analysis, Institute of Mathematics, Faculty of Sciences, \\ Budapest University of Technology and Economics, \\ Egry J. u. 1., H-1111 Budapest, Hungary \\ jtoth@math. bme.hu
}

\begin{abstract}
Computational modeling by integrating compartmental neural technique and detailed kinetic description of pharmacological modulation of transmitter - receptor interaction is offered as a method to test the electrophysiological and behavioral effects of putative drugs. Even more, an inverse method is suggested as a method for controlling a neural system to realize a prescribed temporal pattern. Generation and pharamcological modulation of theta rhytm related to anxiety is analyzed. Integrative modeling might help to find positive allosteric modulators of $\mathrm{GABA}_{\mathrm{A}} \alpha_{1}$ subunits as potential candidates for being selective anxyolitics.
\end{abstract}

Systems Biology is an emergent movement to combine system level description with microscopic details. It might be interpreted as the renaissance of cybernetics [3] and of system theory [4], materialized in the works of Robert Rosen [5]. (For an excellent review on applying the system theoretical tradition to the new systems biology see [6]).

To have a system-level understanding of biological systems [1]2] we should get information from five key features:

- function,

- architecture,

- dynamics,

- control,

- design.

* Thanks to Global Partnership to sponsor JT's visit to Kalamazoo College. We benefited fromDiscussions with Jean-Pierre Rospars (JT), and Ildiko Aradi (PE). Thanks for the motivation and experimental data to Mihály Hajos (Department of Neuroscience, Pfizer, Groton) and to Tamás Kiss, Gergõ Orbán and Balázs Ujfalussy, who made the lion share of the model building and testing both in Kalamazoo and Budapest/Csillebérc. Partial support of the National Scientific Research Council (Hungary) (Nos. T037491, T047132) are also acknowledged by JT. PE thanks the Henry R. Luce Foundation the general support.

M. De Gregorio et al. (Eds.): BV\&AI 2005, LNCS 3704, pp. 1-14 2005.

(C) Springer-Verlag Berlin Heidelberg 2005 
Function. From proteins via genes, cells and cellular networks to the function of our body and mind.

Architecture. From network of gene interactions via cellular networks to the modular architecture of the brain.

Dynamics. Dynamical system theory offers a conceptual and mathematical framework to describe spatiotemporal patterns of concentrations of biochemical components, cellular activity, global dynamical activities (such as measured by electroencephalogram, EEG). Bifurcation analysis and sensitivity analysis reveal the qualitative and quantitative changes in the behavior of the system.

Control. There are internal control mechanisms which maintain the function of the system, while external control (such as chemical, electrical or mechanical perturbation) of an impaired system may help to recover its function.

Design. There are strategies to modify the system architecture and dynamics to get a desired behavior at functional level. A desired function may be related to some "optimal temporal pattern".

While Systems Biology is now generally understood in a somewhat restricted way for proteins and genes, its conceptual and mathematical framework could be extended to neuroscience, as well. Trivially, there is a direct interaction between molecular and mental levels: chemical drugs influence mood and state of consciousness. "Almost all computational models of the mind and brain ignore details about neurotransmitters, hormones, and other molecules." [7].

In this paper we show how to realize the program of Systems Biology in the context of a new, dynamic neuropharmacology. Also, we offer a methodology to integrate conventional neural models with detailed description of neurochemical synaptic transmission in order to develop a new strategy for drug discovery. The procedure is illustrated on the problem of finding selective anxiolytics.

First, we briefly review the functional aspects of our system to be investigated, namely the neuropsychology of anxiety. The septohippocampal system is known to be involved in anxiety. Second, the architecture of the real and the model skeleton network of the septohippocampal system are discussed. Third, since there seems to be a positive correlation between the theta rhythm (i.e. the dynamics of the system), and the level of anxiety, the mechanism of theta rhythm generation is reviewed. Fourth, we review the available data on $\mathrm{GABA}_{\mathrm{A}}$ receptor kinetics to be integrated to the septohippocampal network.

Finally, we conceptually formulate the inverse problem to have a method for design. Having sufficient data for building a detailed kinetic model, we should be able to give advice to drug designers pointing out which subprocess should be modulated to obtain a desired behavior. The specific goal we are focusing on now is to design anxiolytic drugs acting on the $\alpha_{2}$ subunit of $\mathrm{GABA}_{\mathrm{A}}$ receptors without effecting $\alpha_{1}$ subunits related to sedative and hypnotic effects.

\section{Function: Anxiety vs Mood Regulation}

"Anxiety is a complex combination of the feeling of fear, apprehension and worry often accompanied by physical sensations such as palpitations, chest pain and/or shortness of 
breath. It may exist as a primary brain disorder or may be associated with other medical problems including other psychiatric disorders.

A chronically recurring case of anxiety that has a serious affect on your life may be clinically diagnosed as an anxiety disorder. The most common are Generalized anxiety disorder, Panic disorder, Social anxiety disorder, phobias, Obsessive-compulsive disorder, and posttraumatic stress disorder..." [11]

While the historically used mood regulators acting on the barbiturate or benzodiazepine sites of GABA receptors, these drugs have both anxiolytic and hypnotic activity. They enhance the action of GABA via an action at separate binding sites of the $\mathrm{GABA}_{\mathrm{A}}$ receptor.

(Both barbiturates and benzodiazepines shift the GABA concentration-response curve to the left, but barbiturates also increase the maximum response. They act on different states, consequently they have different kinetic effects: average open time of the channel, but not the channel opening frequency is increased significantly by barbiturates. As opposed to benzodiazepines, barbiturate receptors do not contain $\gamma$ subunits (see later). One more difference is that at high concentration GABA receptor channels can directly be opened by barbiturates. For a summary see [45]. Anxiolytic activity was not a particular disadvantage when these drugs were used as hypnotics, hypnosis was a definite disadvantage when they were used as anxiolytics. Recent discoveries made possible the separation between hypnotic and anxyolitic activity and selective hypnotic agents (e.g. zolpidem) are already on the market. Selective anxiolytics are on the preclinical and/or in clinical trial stage.

\section{Architecture: The Septohippocampal Skeleton Network}

It was demonstrated (see e.g. the seminal book of Gray and McNaughton [12] that the septohippocampal system is strongly involved in anxiety and related disorders.

In a joint pharmacological and computational work [13]14] effects of the injection of the positive and negative $\mathrm{GABA}_{\mathrm{A}}$ allosteric modulators diazepam and FG-7142, respectively, were studied. To investigate the dynamical and functional effects of different pharmacological agents by computational tools a skeleton model of the septohippocampal system was established.

The skeleton network model (Fig.11) of the hippocampal CA1 region and the septal GABAergic cells consisted of five cell populations. The hippocampal CA1 pyramidal cells model was a multicompartmental model modified from [17] and supplemented with hyperpolatization activated current $I_{\mathrm{h}}$ based on [18]. Besides $I_{\mathrm{h}}$ the cell model contained sodium $\left(I_{\mathrm{Na}}\right)$, delayed rectifier potassium $\left(I_{\mathrm{K}}\right)$, A-type potassium $\left(I_{\mathrm{K}(\mathrm{A})}\right)$, muscarinic potassium $\left(I_{\mathrm{K}(\mathrm{M})}\right)$, C-type potassium $\left(I_{\mathrm{K}(\mathrm{C})}\right)$, low threshold calcium $\left(I_{\mathrm{Ca}}\right)$ and calcium concentration dependent potassium $\left(I_{\mathrm{K}(\mathrm{AHP})}\right)$ currents. Active and leakage currents were described using the Hodgkin - Huxley formalism. For online supplementary materials, see: http: //geza.kzoo.edu/theta/theta.html.

In the hippocampal CA1 region basket neurons and two types of horizontal neurons were taken into account. Basket neurons formed the fast spiking neuron population of the pyramidal layer, containing $I_{\mathrm{Na}}$ and $I_{\mathrm{K}}$ currents. These model neurons were previously used in [20|21] to account for the population of fast, regularly spiking neurons. 


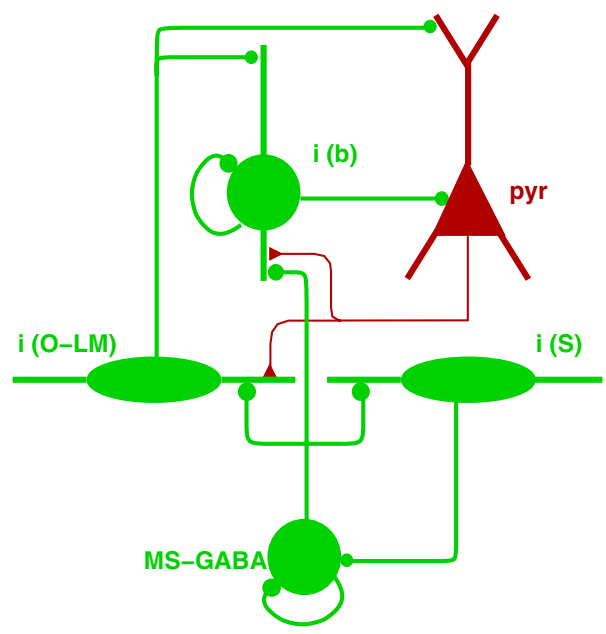

Fig. 1. Left: Computer model of the hippocampal CA1 circuitry. Neuron populations hypothesised to be responsible for the generation of theta oscillation are shown (pyr - pyramidal cells; $\mathrm{i}(\mathrm{O}-\mathrm{LM})$ - horizontal cells projecting to the distal dentrites of pyramidal cells in the lacunosum moleculare layer; $i(b)$ - basket interneurons; $i(S)$ - septally projecting hippocampal horizontal interneurons; MS-GABA - septal GABAergic cells, triangles denote excitatory, dots inhibitory synapses). Connections originating and ending at the same population denote recurrent innervation.

The two types of horizontal neurons represented those interneuron populations whose somata resided at the oriens/alveus border [19]. These neurons were described by the same set of equations as their observed physiological properties are similar and contained sodium, potassium, a high-threshold calcium and hyperpolarization-activated currents [29]. The basket and O-LM neurons were able to generate repetitive action potentials autonomously, and O-LM neurons showed adaptation and low-frequency autonomous firing in the theta band.

Medial septal GABAergic neurons were previously described using single compartment models by Wang [19]. This cell type evokes action potentials repeatedly in clusters. Between any two clusters the cell exhibits subthreshold oscillation but no action potentials due to a slowly inactivating potassium current, which was added to this model neuron besides the Hodgkin - Huxley type sodium and potassium currents.

Connections within and among cell populations were created faithfully following the hippocampal structure. The main excitatory input to horizontal neurons is provided by the pyramidal cells via AMPA (alpha-amino-3-hydroxy-5-methyl-4isoxazolepropionic acid) mediated synapses [22]. Synapses of the septally projecting horizontal cells [25] and synapses of the O-LM cell population innervating distal apical

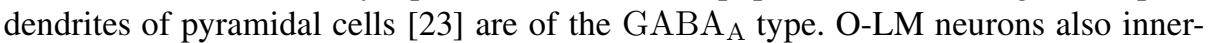
vate parvalbumin containing basket neurons [24]. Basket neurons innervate pyramidal cells at their somatic region and other basket neurons [27] as well. Septal GABAergic cells innervate other septal GABAergic cells and hippocampal interneurons [26 28] (Figure 1). 


\section{Dynamics: Generation of Theta Rhytms}

Theta frequency oscillation of the septohippocampal system has been considered as a prominent activity associated with cognitive function and affective processes. It is well documented that anxiolytics and hypnotics reduce amplitude of septohippocampal oscillatory theta activity, which contributes to their therapeutic effect but causes unwanted side effects, e.g. cognitive impairment as well [16]15].

This detailed, realistic model was used to examine the generation and control of theta oscillation in the hippocampal CA1 region. As shown on Figure $2(A)$, firing of neurons of the four populations were not evenly distributed in time, but time intervals in which firing was significantly reduced were alternated by intervals where enhanced firing was observed. This synchronized state of neural firing was further confirmed by the field potential, which exhibited a prominent $\approx 5 \mathrm{~Hz}$ oscillation as reflected in the power spectrum (Figure2 $(B)$ ).

Simulation results showed that key components in the regulation of the population theta frequency are membrane potential oscillation frequency of pyramidal cells, strength of pyramidal cell-O-LM cell innervation and strength of recurrent basket cell connections. Membrane potential oscillation of pyramidal cells is determined by their averages, passive membrane parameters and parameters of the active currents. Average depolarization in our model results from septal cholinerg innervation. An important

A

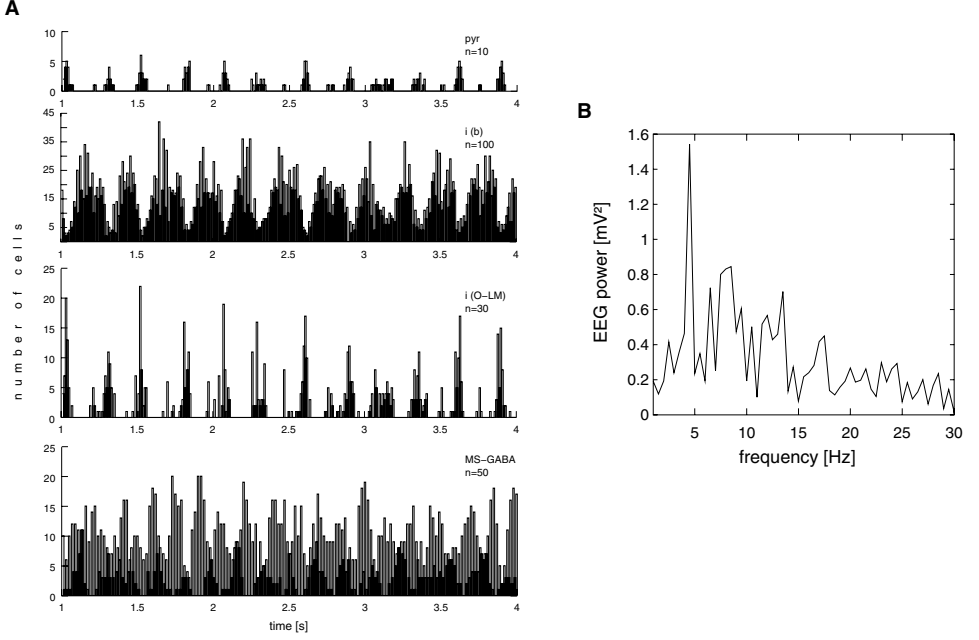

Fig. 2. Appearance of theta frequency population activity in the firing of cells and the Fourier spectrum of the field potential. A, firing histograms were calculated by binning firings of all cells of one of the four populations (pyr - pyramidal cells, i(b) - basket cells, i(O-LM) - orienslacunosum moleculare interneurons, MS-GABA - septal GABAergic cells) into discrete bins. Resulting graph shows the total activity of the respective population. $B$, power spectrum of the field potential. Theta frequency population activity is reflected by temporal modulation of firings in $(A)$ and the $\approx 5 \mathrm{~Hz}$ peak in the power spectrum $(B)$. 
factor is the presence and maximal conductance of the hyperpolarization activated current. If $I_{\mathrm{h}}$ is present it shortens response times of pyramidal cells to hyperpolarizing current pulses and more importantly decreases its variance: $I_{\mathrm{h}}$ acts as a frequency stabilizer. Synaptic strengths in our description are set by convergence numbers and maximal synaptic conductances.

An explanation of intrahippocampal theta oscillation generation-based on this model—includes $i$, signal propagation in the pyramidal cell $\rightarrow$ O-LM cell $\rightarrow$ basket cell $\rightarrow$ pyramidal cell feed-back loop, $i i$, synchronization of neural activity via the recurrent, inhibitory $\mathrm{GABA}_{\mathrm{A}}$ connections within the basket cell network and iii, synchronization of pyramidal cell firing due to rebound action potential generation. It is that the propagation of a single signal throughout this trisynaptic loop would not require the amount of time characteristic to the theta oscillation $(\approx 0.2-0.25 \mathrm{sec})$, thus in the present case the population oscillation is created not by the propagation of single signals but rather the propagation of a "synchronized state" in the network. The observed periodic population activity is brought about by alternating synchronization and desynchronization of cell activities due to the interplay of the above mentioned synchronizing forces and some desynchronizing forces (such as heterogeneity of cell parameters and diversity of synaptic connections), as observed in previous works [21|30].

\section{Control: Integrating GABA Receptor Kinetics to the Receptor Model}

\subsection{Pharmacological Elements}

Receptor Structure. $\mathrm{GABA}_{\mathrm{A}}$ receptors are pentameric structures consisting of multiple subunits. At this moment [31] nineteen subunits have been cloned from mammalian brain. According to their sequence similarities, they have been grouped into seven families: $\alpha, \beta, \gamma, \delta, \epsilon, \pi$ and $\theta$. Only a few dozen among the many combinatorial possibilities exist. The most frequent subtyes two $\alpha$, two $\beta$ and one $\gamma$ subunits. The structural variations imply functional consequnces [31], among others for the kinetic properties.

Drug-Receptor Interaction. A drug/substance may have affinity for the receptor: it may have the capacity to maintain contact with or bound to receptor. Potency is the the absolute number of molecules of drug required to elicit response. Efficacy is the maximum effect obtainable. Therapeutic index: LD50/ED50; the larger it is the safer the drug is.

All the substances binding to any part of the $\mathrm{GABA}_{\mathrm{A}}$ receptor, except GABA, will be called modulators below.

Agonists: Chemicals to open or to facilitate opening the $\mathrm{Cl}^{-}$channels thereby enhancing or creating the inhibitory actions. These are also termed as positive allosteric modulators.

- Endogeneous agonist: the GABA itself.

- Full agonists: of the benzodiazepine family with sedative effects: e.g. diazepam, zolpidem.

- Partial agonists: e.g. bretazenil. 
Inverse Agonists: Chemicals to close or to inhibit opening the $\mathrm{Cl}^{-}$channels (e.g. ) thereby decreasing the inhibitory actions. These are also termed as negative allosteric modulators.

- Full inverse agonist of the benzodiazepine type with anxiogenic effect: e.g. FG-7142.

- Partial inverse agonists: e.g.

Antagonists: Compounds which bind but have no effect on GABA inhibition. They have affinity, but no efficacy, e.g. bicuculline.

1. Desenzitization

- prolonged/continuous use of agonist,

- inhibition of degradation or uptake of agonist,

- cell may attempt to bring its response back to normal by decreasing the number of receptors or binding affinity of receptors.

2. Senzitization

- prolonged/continuous use of receptor blocker,

- inhibition of transmitter synthesis or release,

- cell may attempt to bring its response back to normal by increasing the number of receptors or binding affinity of receptors.

\subsection{The Conventional Tool of Computational Neuroscience}

One way to describe synaptic transmission is to use a gating variable similar to the well known Hodgkin-Huxley formalism:

$$
\begin{aligned}
I_{\text {syn }} & =\bar{g}_{\text {syn }} s\left(V-E_{\text {syn }}\right) \\
\frac{\mathrm{d} s}{\mathrm{~d} t} & =\alpha F\left(V_{\text {pre }}\right)(1-s)-\beta s \\
F\left(V_{\text {pre }}\right) & =\frac{1}{1+\exp \left(\frac{V_{\text {pre }}-\Theta_{\text {syn }}}{K}\right)}
\end{aligned}
$$

with $I_{\text {syn }}$ being the synaptic current, $\bar{g}_{\text {syn }}$ the maximal synaptic conductance, $s$ the gating variable of the synaptic channel, $E_{\text {syn }}$ the synaptic reversal potential, $F(\cdot)$ is an activation function, $\alpha$ and $\beta$ rate functions describing opening and closing of the gate of the synaptic channel, $\Theta_{\text {syn }}$ is a threshold.

Figure 3 illustrates the general form of effects of $\mathrm{GABA}_{\mathrm{A}}$ receptor modulators.

\subsection{An Intermediate Level Strategy: The Pharmacokinetic - Pharmacodynamic Approach}

A theoretical framework with intermediate complexity based on pharmacokinetics pharmacodynamics (PK/PD) was suggested to model the effects of GABA modulators on EEG in a series of papers [32[33]. Pharmacokinetics generally is supposed to describe drug disposition and biophase equilibration, diffusion included. In the applied 


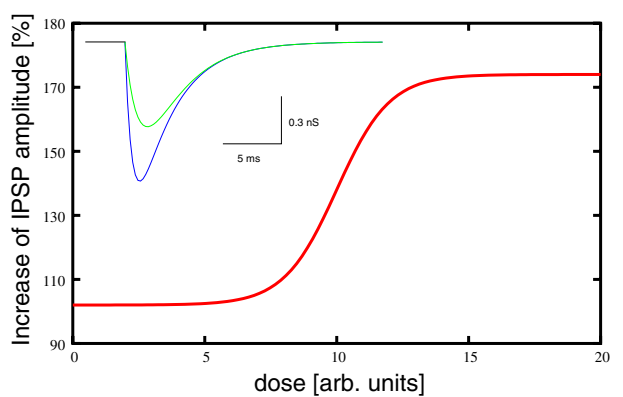

Fig. 3. Modelling the effects of allosteric $\mathrm{GABA}_{\mathrm{A}}$ receptor modulators. In a simple description of synaptic transfer the strength of synapses was modulated via the $\bar{g}_{\text {syn }}$ parameter in eq. (1a) in a dose dependent manner. Inset: modelled inhibitory postsynaptic potentials before (smaller amplitude) and after (larger amplitude) administration of positive $\mathrm{GABA}_{\mathrm{A}}$ allosteric modulator.

framework pharmacodynamics might consist of two stages: one for drug-receptor interaction, and another one for the signal transduction processes or stimulus-response relationship. The stimulus - response function is empirically determined, and intentionally neglects the architecture of the system under investigation. While this approach proved to be an efficient method, we believe that the architecture of the neural circuits should be taken into account explicitly to get a better understanding of the underlying neural mechanisms.

\subsection{Kinetic Modeling of $\alpha_{1}$ and an $\alpha_{2}$ Modulators: A Plan}

From Pharmacodynamics to Detailed Kinetic Scheme. A more effective, but certainly most expensive, modelling tool to evaluate the pharmacological effects of the different modulators, or even to give help for offering new putative molecules for drug discovery, is the inclusion of more detailed kinetic studies of GABA receptor modulation.

Suppose the dose response curve of $\mathrm{GABA}_{\mathrm{A}}$ is given and we also have the dose response curve of a modulator or a drug-modulator pair. Then, one can draw a few qualitative consequences.

It is important to fix, if the effect is measured as a function of drug concentration which is usually a hyperbola (naturally, without any inflexion point), or, as a function of the logarithm of the concentration in which case again a saturation curve is obtained but with an inflexion point at $\mathrm{ED}_{50}$.

The effect of different modulators is as follows. If the effect is that the saturation point (the limit of the dose response curve atinfinite modulator concentration) is smaller then without the modulator, then the modulator is a partial agonist. If the modulator has no effect (although it binds to the same binding site or to a site which hinders the endogenous agonist to act), i.e. the dose effect curve is constant zero, then we have an antagonist. If the effect of the modulator is a monotonously decreasing curve then we have an inverse agonist. One may also have a dose response curve shifted to the right (left); the modified system (modulator, or modulator + endogeneous agonist) has 
a smaller (larger) potency, i.e. a larger (smaller) number of drug molecules are required to elicit the same response. If the modified system's curve goes parallel with the original but below it (i.e. not only its limit is smaller), then the efficacy is decreased.

Kinetic Schemes. Jones and Westbrook [8] established a model for describing the rapid desensitization of the $\mathrm{GABA}_{\mathrm{A}}$ receptors. More specific kinetic models should be studied to describe the effects of the different (full and partial) agonists and antagonists. Baker et al. [34] explained the functional difference between the effects of protophol (which has hypnotic effect) and of midazolam (a sedative - amnestic drug) based on a detailed kinetic model.

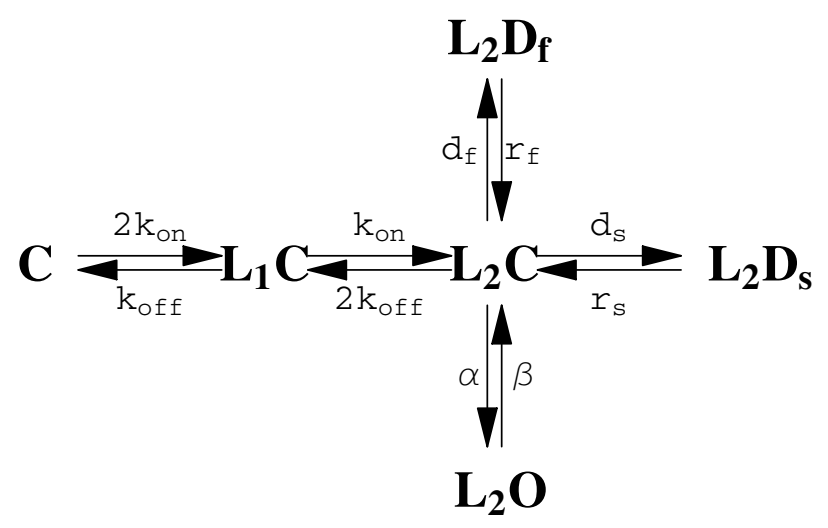

Fig. 4. Basic scheme of GABA $A_{\mathrm{A}}$ receptor kinetics. $C, L_{1} C, L_{2} C$ denote closed states with zero, one and two bound ligands respectively. $L_{2} O$ is the open state, while $L_{2} D_{f}, L_{2} D_{s}$ are the desenzitized states. Modulators may effect different steps of these complex chemical reaction.

The main difference is that protophol modifies the desenziation processes, more dramatically the slow desenzitation steps and the modified kinetic parameters. These differences imply distinct behavior of the network (synchronization, frequency of oscillation) and therefore also in function.

\subsection{Models of Anxioselective Actions: Search for Data}

Recently it became clear that $\alpha$ subunits exhibit a remarkable functional specificity. Genetic manipulations helped to show that $\alpha_{1}$ subunits are responsible for mediating sedative effects, while $\alpha_{2}$ subunits mediates anxiolytic effects [10]. Preliminary experimental data and modelling studies for the the effects of the preferential GABA $\alpha_{1}$ and $\alpha_{2}$ positive allosteric modulator, zolpidem and $L 838,417$ for the septohippocampal theta activity have been reported [9].

In this study we examined the effects of the $\alpha_{1}$ and $\alpha_{2}$ subtype-selective benzodiazepine site ligand zolpidem and $L 838,417$ on the septohippocampal system. In electrophysiological experiments extracellular single unit recordings were performed from 
medial septum/diagonal band of Broca with simultaneous hippocampal (CA1) electroencephalogram (EEG) recordings from anesthetized rats. Both of the drugs eliminated the hippocampal theta oscillation, and turned the firing pattern of medial septal cells from periodic to aperiodic, but only the zolpidem reduced the firing rate of the these neurons. In parallel to these experimental observations, a computational model has been constructed to clearly understand the effect of these drugs on the medial septal pacemaker cells. We showed that the aperiodic firing of hippocampo-septal neurons can reduce the periodicity of the medial-septal cells, as we have seen in the case of the $L 838,417$. The reduction of firing rates in the case of zolpidem is attributed to the increase of the synaptic conductances and the constant inhibition of these cells. We modelled these drug effects by modifying (i) the synaptic maximal conductances of the GABA synapses. (ii) the constant excitatory drive of the median septal cells and (iii) the hippocampal input. The incorporation of a more detailed synaptic model is in progress.

Zolpidem increases by concentration-dependent manner the duration and amplitude of the postsynaptic current, most likely by enhancing the affinity of the receptors for GABA [35]. It significantly increased the amplitude and frequency of the postsynaptic current, but these effects were diminished or absent in neurons from $\alpha_{1}$ knock-out mice [36].

There seem to be compounds, which might have comparable binding affinity but different efficacies at the various subtypes, thereby preferentially exerting its effects at subtypes thought to be associated with anxiety. $L 838,417$ seems to be an an example for efficacy selective compounds [37], but kinetic or even pharmacodynamic data could not be found (at least not very easily) in the public domain.

\subsection{Modulation of Synaptic and Extra-Synaptic GABA $A_{A}$ Receptors}

There are different mechanisms for postsynaptic modulation. It might be a long-term change in the number of receptors, a change in the affinity of a ligand, or a change on ionic conductances [38]. Recently it was emphasized that in addition to the conventional ("phasic") synaptic transmission the extrasynaptic "tonic" GABAergic cell-cell communication also has a significant functional role [39|40|31]. GABA can activate receptors on presynaptic terminals or at neighboring synapses ('spillover'). The phasic and tonic inhibitions are spatially and temporally discrete, and continuous, respectively. (For a review on non-synaptic communication see [46].) The two distinct mechanisms of the $\mathrm{GABA}_{\mathrm{A}}$-receptor mediated inhibition implies different functional roles. Also, most likely different receptor subtypes mediate the two types of inhibition, and might be modulated by different kinetic schemes. Future works will show the similarities and differences among the different kinetic schemes behind the modulatory mechanisms of the phasic and tonic inhibition.

\subsection{Direct Problem: To Simulate Modulatory Effects}

Kinetic modeling of synaptic transmission has a flexibility in the level of detailed description from chemical kinetic to simplified representation [41]. The development of new pharmacological, electrophysiological and computational techniques make possible to investigate the modulatory effects of putative drugs for synaptic currents, and 
consequently for local field potentials and even behavioral states. Putative drugs with given kinetic properties can be tested in silico before (instead of?) real chemical and biological studies.

\section{Design (Inverse Problem): From System Identification to Optimal Temporal Patterns}

We have shown that in a moderately complex conductance-based model of the hippocampal CA1 region theta rhythm generation can be observed and major interactions between cell populations and within cells responsible for the phenomena can be identified. These results qualify the model for consideration as a useful tool in the hands of pharmacologists, physiologists and computational neuroscientists to complete their repertoire of available tools in the search for efficient and specific drugs.

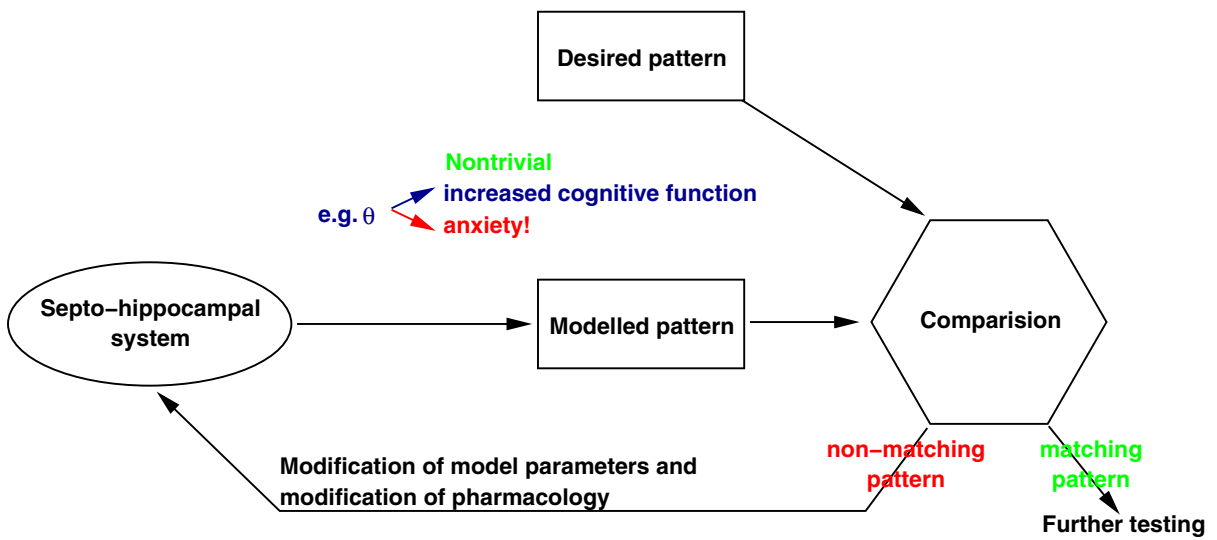

Fig. 5. Computational neuropharmacology—an idealized method for drug discovery. See text for a description.

Figure 5 is an oversimplified scheme offered for finding finding a modulator to set optimal septohippocampal EEG pattern.

In order to decrease anxiety first a desired EEG pattern shold be defined. Anxyolitics should reduce the amplitude the theta amplitude (but preserving the cognitive performance and avoiding sedative hypnotic side effects). Computational analysis should offer a best kinetic scheme and rate constant to modulate te fixed network to minimize the deviation from the desired "optimal pattern". (Network architecture is supposed to be fixed. By neglecting this assumption we should turn from neuropharmacology to neurosurgery...) Most likely there are more than one possibilities to reach the goal, and model discrimination and parameter estimation techniques may help to narrowing the alternatives.

As it is known from chemical kinetics [43147] sensitivity analysis shows that in a kinetic scheme there are "more and less important" components and reactions. It helps 
to answer the question, whether how to modify the structure of a given drug to change the reaction rate constants in the desired direction-and leaving everything else intact.

\section{Discussion, Further Research}

The aim of the present paper is to offer conceptual and mathematical frameworks to integrate network and receptor level descriptions for investigating the effects of potential drugs for the global electrical patterns of a neural center, and and for the behavioral states (mood, consciousness etc.). Once we have understood (i) the basic mechanisms of rhythm generation, (ii) the elementary steps of the modulatory process, we shall a be able to give advice to drug designers pointing out which subprocess and how to be modulated to reach a given goal.

Specifically, we briefly reviewed some aspects of $\mathrm{GABA}_{\mathrm{A}}$ receptor kinetics, and the effects of (full and partial) agonists, antagonists and inverse antagonists to septohippocampal theta rhytms. The specific goal we are focusing is to design anxiolytic drugs with as small as possible side effects. While is is known that positive allosteric modulators acting on $\mathrm{GABA}_{\mathrm{A}} \alpha_{1}$ subunits are potential candidates for being selective anxyiolitics, integrative computational modeling would help to find the appropriate kinetic properties of potential drugs.

\section{References}

1. Kitano H.: Systems biology: a brief overview Science 295 2002) 1662-1664

2. Csete ME., Doyle JC.: Reverse Engineering of Biological Complexity. Science, 295 (2002) 1664-1669

3. Wiener N: Cybernetics: or Control and Communication in the Animal and the Machine. MIT Press, 1948.

4. Von Bertalanffy L: General Systems Theory: Foundations, Development, Applications. George Braziller, New York/NY, 1969

5. Rosen R: Anticipatory Systems. Philosophical, Mathematical and Methodological Foundations. Pergamon Press, 1985

6. Wolkenhauer O: Systems biology: The reincarnation of systems theory applied in biology? Briefings in Bioinformatics, 2 (2001) 258-270

7. Thagard, P. : How molecules matter to mental computation. Philosophy of Science, 69 (2002) 429-446.

8. Jones B, Westbrook GK Desensitized states prolong gabaa channel responses to brief agonist pulses. Neuron, 15 (1995) 181-191.

9. Ujfalussy B, Orbán G, Kiss T, Hoffmann WE, Érdi P, and Hajós M" Anxiolytics and septohippocamapal oscillation: Pharmacological and computational analysis of action of gaba ${ }_{a} \alpha_{1}$ and $\alpha_{2}$ receptor allosteric modulators. Conf. Hungarian Neuroscience Association, abstract, 2005.

10. Rudolph $\mathrm{U}$ and Mohler $\mathrm{H}$. Analysis of gaba $a$ receptor function and dissection of the pharmacology of benzodiazepines and general anesthetics through mouse genetics. Annu. Rev. Pharmacol. Toxicol., 44, 475-498, 2004.

11. From Wikipedia, the free encyclopedia. Anxiety. http://en.wikipedia.org/wiki/Anxiety

12. Gray, J.A., McNaughton, N., (2000) The Neuropsychology of Anxiety: An Enquiry into the Functions of the Septo-hippocampal System, $2^{\text {nd }}$ ed., Oxford University Press, Oxford 
13. Hajós M, Hoffmann WE, Orbán G, Kiss T, and Érdi P.: Modulation of septo-hippocampal $\theta$ activity by $\mathrm{GABA}_{\mathrm{A}}$ receptors: An experimental and computational approach. Neuroscience, 126(3):599-610, 2004.

14. Kiss T, Orbán G, Érdi P.: Modelling hippocampal theta oscillation: applications in neuropharmacology and robot navigation. International Journal of Intelligent Systems (in press)

15. Lees G and Dougalis A.: Differential effects of the sleep-inducing lipid oleamide and cannabinoids on the induction of long-term potentiation in the cal neurons of the rat hippocampus in vitro. Brain Research, 997(1):1-14, 2004.

16. A Maubach K, Martin K, Choudhury HI, and Seabrook GR.: Triazolam suppresses the induction of hippocampal long-term potentiation. Neuroreport, 15(7):1145-1149, 2004.

17. Varona P, Ibarz M, López-Aguado L, and Herreras O.: Macroscopic and subcellular factors shaping population spikes. Journal of Neurophysiology, 83:2192-2208, 2000.

18. Magee JC.: Dendritic hyperpolarization-activated currents modify the integrative properties of hippocampal CA1 pyramidal neurons. Journal of Neuroscience, 18(19):7613-7624, October 1998.

19. Wang XJ.: Pacemaker neurons for the theta rhythm and their synchronizati on in the septohippocampal reciprocal loop. Journal of Neurophysiology, 87(2):889-900, 2002.

20. Wang XJ and Buzsáki G.: Gamma oscillation by synaptic inhibition in a hippocampal interneuron network model. J Neurosci, 16:6402-6413, 1996.

21. Orbán G, Kiss T, Lengyel M, and Érdi P.: Hippocampal rhythm generation: gamma-related theta-frequency resonance in CA3 interneurons. Biol Cybern, 84(2):123-132, 2001.

22. Lacaille JC, Mueller AL, Kunkel DD, and Schwartzkroin PA.: Local circuit interactions between oriens/alveus interneurons and ca1 pyramidal cells in hippocampal slices: electrophysiology and morphology. J Neurosci, 7:1979-1993, 1987.

23. Lacaille, J.C., Williams, S. (1990) Membrane properties of interneurons in stratum oriensalveus of the CA1 region of rat hippocampus in vitro. Neuroscience 36:349-359.

24. Katona I., Acsády L., Freund T.F. (1999) Postsynaptic targets of somatostatinimmunoreactive interneurons in the rat hippocampus. Neuroscience 88:37-55.

25. Jinno $S$ and Kosaka T.: Immunocytochemical characterization of hippocamposeptal projecting gabaergic nonprincipal neurons in the mouse brain: a retrograde labeling. Brain Res, 945:219-231, 2002.

26. Freund TF and Antal M.: Gaba-containing neurons in the septum control inhibitory interneurons in the hippocampus. Nature, 336:170-173, 1988.

27. Freund TF and Buzsáki G.: Interneurons of the hippocampus. Hippocampus, 6:347-470, 1996.

28. Varga V, Borhegyi Zs, Fabo F, Henter TB, and Freund TF. In vivo recording and reconstruction of gabaergic medial septal neurons with theta related firing. Program No. 870.17. Washington, DC: Society for Neuroscience., 2002.

29. Maccaferri G and McBain CJ:. The hyperpolarization-activated current (Ih) and its contribution to pacemaker activity in rat CA1 hippocampal stratum oriens-alveus interneurones. Journal of Physiology, 497:119-130, 1996.

30. Kiss T., Orbán G., Lengyel M., Érdi P. (2001) Intrahippocampal gamma and theta rhythm generation in a network model of inhibitory interneurons. Neurocomputing 38-40:713-719.

31. Farrant, M. and Nusser, Z.: Variations on an inhibitory theme: phasic and tonic activation of GABAA receptors. Nature Reviews Neuroscience. 6, 215-229. 2005.

32. Visser SA, Smulders CJ, Reijers BP, Van der Graaf PH, Peletier LA, Danhof M.: Mechanismbased pharmacokinetic-pharmacodynamic modeling of concentration-dependent hysteresis and biphasic electroencephalogram effects of alphaxalone in rats. J. Pharmacol Exp Ther. 302(3):1158-67. 2002 
33. Visser SA, Wolters FL, Gubbens-Stibbe JM, Tukker E, Van Der Graaf PH, Peletier LA, Danhof M.: Mechanism-based pharmacokinetic/pharmacodynamic modeling of the electroencephalogram effects of GABAA receptor modulators: in vitro-in vivo correlations. $J$ Pharmacol Exp Ther. 2304(1):88-101. 2003

34. Baker PM, Pennefather PS, Orser BA, Skinner FK.: Disruption of Coherent Oscillations in Inhibitory Networks With Anesthetics: Role of GABAA Receptor Desensitization J. Neurophysiol. 88:2821-33. 2002

35. Perrais D, Ropert N: Effect of Zolpidem on Miniature IPSCs and Occupancy of Postsynaptic GABAA Receptors in Central Synapses. J. Neuroscience, 19:578-588. 1999

36. Goldstein PA, Elsen FP, Ying SW, Ferguson C, Homaincs GE, Harrison NE: Prolongation of Hippocampal Miniature Inhibitory Postsynaptic Currents in Mice Lacking the GABAA Receptor $\alpha_{1}$ Subunit. Neurophysiol 88: 3208-3217, 2002;

37. Atack, J. R.: Anxioselective Compounds Acting at the GABAA Receptor Benzodiazepine Binding Site. Curr Drug Target CNS Neurol Disord. 2.4 (Aug) (2003) 213-232

38. Cooper JR, Bloom FE and Roth RH: The Biochemcial Basis of Neuropharamcology., 8 th edition, Oxford University Press 2003.

39. Mody I: Distinguishing between GABA(A) receptors responsible for tonic and phasic conductances. Neurochem Res 26:907-913.

40. Mody, I., and Pearce, R.A.: Interneuron diversity series: Diversity of inhibitory neurotransmission through GABAA receptors. Trends in Neuroscience 27:569-575 (2004).

41. Destexhe, A., Mainen, Z. A., Sejnowski, T. J.: Kinetic Models of Synaptic Transmission. In: Koch, C., Segev, I. (eds.): Methods in Neuronal Modeling (2nd ed). MIT Press, Cambrdige, MA (1998) 1-30

42. Deupree, J. D., Bylund, D. B.: Basic Principles and Techniques for Receptor Binding. Tocris Review 18 (March) (2002)

43. Érdi, P., Tóth, J.: Mathematical Models of Chemical Reactions. Theory and Applications of Deterministic and Stochastic Models. Princeton University Press, Princeton, and Manchester University Press, Manchester (1989)

44. Jones, J. W., Westbrook, G. L.: Desensitized States Prolong GABA A Channel Responses to Brief Agonist Pulses. Neuron 15 (1995) 181-191

45. Martin, I. L., Dunn, S. M.: GABA Receptors. Tocris Review 20 (March) (2002)

46. Vizi ES: Role of High-Affinity Receptors and Membrane Transporters in Nonsynaptic Communication and Drug Action in the Central Nervous System Pharmacol. Rev. 52,63-90. 2000.

47. Turányi, T.: Sensitivity Analysis of Complex Kinetic Systems: Tools and Applications. J. Math. Chem. 5 (1990) 203-248 